\title{
A New Hybrid Algorithm to Solve the Task Scheduling Problem in Grid Computing
}

\author{
Hamid Salehi \\ Department of computer science, \\ Shiraz University, \\ Shiraz,Iran
}

\author{
Reza Boostani \\ Department of computer science, \\ Shiraz University, \\ Shiraz,Iran
}

\author{
Asou Aminnezhad \\ Department of computer science, \\ University Putra Malaysia, \\ Selangor, Malaysia
}

\begin{abstract}
The new generation of networks, distributed systems, grid computing, which allows users to share files and Users need to use different sources to provide. Grid computing system as one of the competing technologies for cloud computing can be considered to have many advantages for users. One goal of grid computing systems, the management of computing resources for processing user applications or clients So that the resulting high quality of service, lower costs and greater flexibility is. In this paper, to solve the scheduling problem in grid computing system combining genetic algorithms and algorithms of gravity is used.
\end{abstract}

\section{General Terms}

Grid scheduling

\section{Keywords}

Grid computing systems, Genetic algorithms, Algorithms gravity

\section{INTRODUCTION}

Grid computing system is the new generation of distributed networks that allows users to share files and provides users need to use different sources. Grid computing system is considered as one of the technologies competing for the supercomputer that can have many benefits for users. Nowadays grid as a model for solving problems in science, engineering, industry and trade are discussed. With the increasing number of users in such problems, the grid computing and storage resources for other needs have been resolved [1].

One goal of the Grid Computing Management of computing resources for processing user applications or clients that lead to high-quality services at lower costs and greater flexibility is [2]. With the increase in grid computing resources to Grady system is felt that to be able to manage this kind of resources to quickly respond to various requests. Therefore, users work schedule for processing by the appropriate resources in grid networks, as a fundamental issue in achieving high performance in grid computing systems have been proposed network. However, the design and implementation of efficient algorithms for scheduling tasks of an application as a problem NP Complete in the network Grid computing is becoming a big challenge [3].

What are the different types and grades into different Barmhasbaty. The objective of Grid scheduler, the optimal assignment of tasks to resources. Today, with the growing importance of the issues to quickly find answers, solve many problems not amenable to traditional methods of random search algorithms over a comprehensive search of the issue will be used. At present innovative algorithms that are used for grid scheduling problem, generally, genetic algorithms, simulated refrigeration, hybrid genetic algorithms and simulated refrigeration, unlawful search, colony ant colony optimization, and the optimization of birds.

GA algorithm to schedule the work of the late 1990s in a heterogeneous computing environment has been used [4] Braun and colleagues conducted a comparison between eleven static algorithms [5]. Their simulations showed that GA consistently obtains the best solution compared to other algorithms. In 2007, Mr. Carretero and colleagues a valuable study on the benefits of GA to design of efficient grid scheduler, with the goal of minimizing Makespan and workflow offered [6]. In 2008, Yuan an improved adaptive algorithm combining GA with neighborhood search is introduced, and simulations show that the proposed algorithm can the performance of scheduling dramatically improve Grid [7].

Fidanova job scheduling algorithm based on SA algorithm is presented that achieves better results as compared to ACO[8]. In 2008, Kazem and his colleagues a modified SA for scheduling independent tasks in Grid environment was provided[9]. Experimental results show that this algorithm is compared with the results of other algorithms in the paper were obtained; it can improve the performance of a static samples. In 2006, Zheng and his colleagues, a parallel algorithm GSA, GA and SA which combines the advantages of the proposed Grid scheduling problem [10].

In this paper, a new hybrid algorithm combining genetic algorithm and algorithm to solve scheduling tasks in the network gravity grid computing is proposed.

Rest of the paper is structured as follows: in Section 2 with a description of the proposed Grid network is presented. Procedures set forth in Section 3 and Section 4 of the proposed algorithm and experimental results are shown comparing the proposed algorithm with Genetic Algorithms. In Section 5 the conclusions expressed.

\section{Description of the problem}

In grid environment is presented in this paper, there are $\mathrm{N}$ sources; each of these sources can be located anywhere in the geographical environment. Each source is composed of a number of machines per machine processor to process data from a number of different perks and a different kind of performance has been established. For example, a car may be a server that contains multiple processors, and each processor has its many perks, it is also a car can be a processor with different performance. In addition, each processor has a different fee for processing information. Processors using the communication channels they are connected with the car. Finally, all of these resources to the grid scheduling system 
are connected through a communication channel. Properties considered in Table 1 for the sources listed.

Table 1. Properties considered for resource

\begin{tabular}{|c|c|c|}
\hline Source & Machine & Processor \\
\hline $\begin{array}{l}\text { Bandwidth used to receive and transmit } \\
\text { data }\end{array}$ & $\begin{array}{l}\text { The operating system of the } \\
\text { machine }\end{array}$ & Power processors based on MIPS \\
\hline \begin{tabular}{|l|} 
Machine list provided by the supplier \\
\end{tabular} & $\begin{array}{l}\text { Machine architecture, such as Intel, } \\
\text { Mac and .... }\end{array}$ & Find the cost of work done per second \\
\hline \multirow[t]{3}{*}{ Geographical area source } & List of avaliable processors & Percent of the perks of working days \\
\hline & & Percent of the processing power on holidays \\
\hline & & $\begin{array}{c}\text { Percent power at other times, such as } \\
\text { midnight }\end{array}$ \\
\hline
\end{tabular}

In this environment, grid users by their requests to different outgoing rate a file system to a Grid scheduler, Grid scheduling system using the scheduling algorithm, the users do the allocation of resources for the processing of deals. Each user for the processing of your actions, proposed completion time and cost of the proposed works. The scheduling algorithm, the users work in minimum possible time and with less cost. After scheduling tasks by scheduling algorithm and their implementation on grid systems, processed information is sent to the user. The parameters considered for application users and their tasks are shown in Table 2.

Table 2. Parameters intended for users and applications work

\begin{tabular}{|l|l|}
\hline \multicolumn{1}{|c|}{ Application tasks } & \multicolumn{1}{|c|}{ User } \\
\hline $\begin{array}{l}\text { Number of work orders requested by the } \\
\text { millions }\end{array}$ & Do list \\
\hline The file size of the requested user & Total processing time for the proposed work \\
\hline $\begin{array}{l}\text { Type of work required operating system } \\
\text { user request }\end{array}$ & Suggested price for the whole process works \\
\hline $\begin{array}{l}\text { Find business application The kind of } \\
\text { architecture requirements }\end{array}$ & User geographical area. \\
\hline & The priority parameter "suggested time" \\
\hline & Priority parameter "price" \\
\hline
\end{tabular}

The system works in a off-line users and is not intended to limit the user's work process. Each processor works on the operating system and its associated processing architecture and the processor does not halt, The CPU time allocated to a task, the processor at the end of the process will work.

\section{The proposed algorithm}

In this paper, to solve the scheduling problem in grid computing system combining genetic algorithms and algorithms of gravity is used. Table -3 of the proposed algorithm on grid computing system model has been implemented. The grid system with two sources, each of which has two machines, and each machine has two processors is to process things.

Table 3. Basic information of resources in Grid computing model.

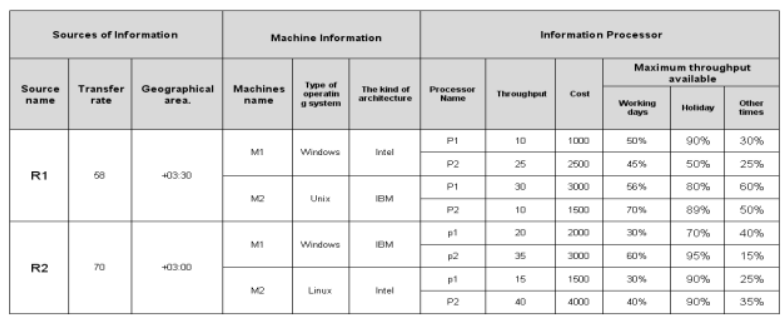

Basic information related to the users are shown in Table 4. In this table there are three users, each user profile to work offline has been sent to the grid scheduling system Samples processed by the CPU to be placed on the grid system.

Table 4. Basic information of users in the Grid computing model

\begin{tabular}{|c|c|c|c|c|c|c|c|c|c|c|c|}
\hline \multicolumn{7}{|c|}{ work the information } & \multicolumn{5}{|c|}{ Information users } \\
\hline $\begin{array}{l}\text { User } \\
\text { name } \\
\text { nome }\end{array}$ & $\begin{array}{c}\text { Transmissi } \\
\text { on rates }\end{array}$ & $\begin{array}{c}\text { Suggested } \\
\text { tee }\end{array}$ & $\begin{array}{c}\text { Suggested } \\
\text { time }\end{array}$ & $\begin{array}{l}\text { Fisst } \\
\text { time } \\
\end{array}$ & $\begin{array}{l}\text { Spending } \\
\text { pitioritizg }\end{array}$ & $\begin{array}{c}\text { Geographi } \\
\text { cal area }\end{array}$ & $\begin{array}{l}\text { Mumber } \\
\text { of work }\end{array}$ & wook size & $\begin{array}{c}\text { Mumber } \\
\text { of orders }\end{array}$ & $\begin{array}{c}\text { Kind } \\
\text { operting } \\
\text { system }\end{array}$ & $\begin{array}{l}\text { Kind of } \\
\text { arcine ettue }\end{array}$ \\
\hline \multirow{3}{*}{ U1 } & \multirow{3}{*}{30} & \multirow{3}{*}{1000} & \multirow{3}{*}{200} & \multirow{3}{*}{7.0} & \multirow{3}{*}{3.0} & \multirow{3}{*}{$+03: 30$} & 1 & 50 & 150 & windows & Intel \\
\hline & & & & & & & 2 & 35 & 80 & Unix & IBM \\
\hline & & & & & & & 3 & 8 & 20 & windows & Intel \\
\hline \multirow{3}{*}{ U2 } & \multirow{3}{*}{45} & \multirow{3}{*}{9000} & \multirow{3}{*}{180} & \multirow{3}{*}{4.0} & \multirow{3}{*}{6.0} & \multirow{3}{*}{$+03: 30$} & 1 & 18 & 50 & Linux & Intel \\
\hline & & & & & & & 2 & 25 & 60 & Unix & IBM \\
\hline & & & & & & & 3 & 43 & 100 & Unix & IBM \\
\hline \multirow{3}{*}{ U3 } & \multirow{3}{*}{60} & \multirow{3}{*}{1500} & \multirow{3}{*}{150} & \multirow{3}{*}{5.0} & \multirow{3}{*}{5.0} & \multirow{3}{*}{$+03: 00$} & 1 & 5 & 15 & windows & Intel \\
\hline & & & & & & & 2 & 20 & 55 & windows & IBM \\
\hline & & & & & & & 3 & 30 & 70 & Linux & Intel \\
\hline
\end{tabular}

In the proposed algorithm to identify the best sources and processors of Table -3 is used for data encoding. Available sources of information for indexing CPU encoding is used, so that the new coded for each of the processors is considered a unique index. For example, an index number in Table II processor machine coded in the source notes And index number four the number of processors in a machine, a second source suggests. Table 5 shows how the coding in Table 3 , each row of the table refers to the processors used in Table 3 .

Table 5. Sources encoded in the grid computing model

\begin{tabular}{|c|c|c|c|}
\hline Number of rows & Resource name & Machines name & CPU Name \\
\hline 0 & R1 & M1 & P1 \\
\hline 1 & R1 & M1 & P2 \\
\hline 2 & R1 & M2 & P1 \\
\hline 3 & R1 & M2 & P2 \\
\hline 4 & R2 & M1 & P1 \\
\hline 5 & R2 & M1 & P2 \\
\hline 6 & R2 & M2 & P1 \\
\hline 7 & R2 & M2 & P2 \\
\hline
\end{tabular}

In the proposed algorithm to identify the best practices of coding work table is used. Each row of the table of user actions referred to in Table 4. The following table (6) how the code works, in Table 4 show.

Table 6. Coding jobs in the Grid computing model

\begin{tabular}{|c|c|c|}
\hline Number of rows & User name & Number of work \\
\hline 0 & U1 & 1 \\
\hline 1 & U1 & 2 \\
\hline 2 & U1 & 3 \\
\hline 3 & U2 & 1 \\
\hline 4 & U2 & 2 \\
\hline 5 & U2 & 3 \\
\hline 6 & U3 & 1 \\
\hline 7 & U3 & 2 \\
\hline 8 & U3 & 3 \\
\hline
\end{tabular}




\subsection{Chromosome Structure}

In the proposed algorithm to display the total number of jobs available in the chromosome of one-dimensional array is used for processing. Chromosome is composed of two fields, each home to $\mathrm{UT}_{\mathrm{i}}$ and $\mathrm{PU}_{\mathrm{i}}$ are shown. $\mathrm{PU}_{\mathrm{i}}$ First field to cpu number $i$, the source table is encoded And second field $U T_{i}$, the $i$, the table refers to the encoding tasks.

\begin{tabular}{|l|l|l|l|l|l|l|l|l|}
\hline 2,0 & 6,1 & 8,7 & 0,0 & 1,3 & 7,4 & 5,2 & 3,6 & 4,3 \\
\hline
\end{tabular}

Figure 2. Structure of the proposed algorithm

chromosomes

As you can see from the structure of chromosomes in Figure 2 , each number in the array, the number of work that must be processed by the CPU shows. For example, the gene content of 1,6 is, in fact, 6 of the table refers to the encoding tasks that should CPU 1, which is encoded in the resource table, to be processed. In the proposed algorithm, each chromosome represents a schedule for all the tasks users.

\subsection{Merit}

Chromosome Merit in the proposed algorithm to reduce the completion time of all tasks to process scheduler in Grid computing systems and improve work processes so that users need to be fully considered by the parties. Users Equation 1 is used to calculate a Merit.

$F_{j}=\left(\frac{C T_{j}}{O P_{j}} * P C_{j}\right)+\left(\frac{T T_{j}}{O T_{j}} * P T_{j}\right)$

In the above equation $P_{j}$, the cost is intended for user $j, \mathrm{OP}_{j}$ represents the total processing cost of the proposed works is the user $\mathrm{j}$, And $\mathrm{CT}_{\mathrm{j}}$ represents the estimated costs to process user jobs on Grid Scheduling System $\mathrm{j}$ and $\mathrm{PC}_{\mathrm{j}}$ indicates priority intended for user $\mathrm{j}$ is the cost.

Also $T_{j}$, is intended for time user $\mathrm{j}, \mathrm{OT}_{\mathrm{j}}$ represents the total processing time of the proposed work is the desired user $\mathrm{j}$ and $\mathrm{TT}_{\mathrm{j}}$, shows the computation time for processing user jobs on Grid Scheduling System $\mathrm{j}$ and $\mathrm{PT}_{\mathrm{j}}$ represent preferences intended user $\mathrm{j}$, for a time.

The merits of a chromosome, the proposed algorithm Merit total completion time of all users and for Grid scheduling system to process all things are considered, Whatever the Merit of the chromosome, the chromosome with the lower Merit is better. Equation 2 is used to calculate the Merit of the chromosome.

Fitness $=\sum_{j=0}^{j} F_{j}$

\subsection{Population Optimization}

At this stage the chromosomes selected for transfer to the next generation by using gravity algorithm improved the percentage of the population of chromosomes as the new children are added to the population.

In the proposed algorithm, the number of chromosomes in a loop selection and ranking algorithms using the gravitational force is called for. Chromosomes in each iteration of the genetic algorithm gravitational force as a solution to current
$\mathrm{CU}$ received and applied to optimize multi-stage operation in which the chromosomes.

The gravitational algorithm proposed method is to first search the problem space neighborhood of the current solution is created The combination of the first two methods are used to calculate the gravitational force, To move in the search space of single and multiple combinations are used.

Algorithms defined in the neighborhood of gravity is to the total solution nominated scanned from beginning to end, And most detailed source of data is processed to act shall be selected, Then run a small operation that has changed are randomly selected and the source. In the end, the best answer to the gravitational algorithm genetic algorithm named Best Solution returns.

\section{Implementation of the consequences}

To test the performance, the proposed algorithm (GAGELS) is compared with the algorithm [GA]. Algorithms are used to implement the programming language C \#. Net 2010. Algorithm on a computer with a processor Intel (R) Pentium (R) $4 \mathrm{CPU} 3.00 \mathrm{GHz}$ and 2.00GB RAM will run. To compare these two algorithms designed 10 sets of test data to the grid system of small, medium and large covers. Each test case where $\mathrm{N}$ represents the number of Test N_DAY test Day to day, and that the test set will be processed in a grid network show, The test data set is shown in Table 7.

Table 7. Data collection was designed to test

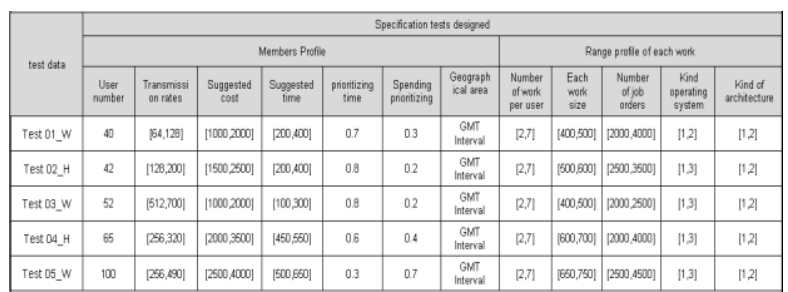

Since each test requires a Model of Table 7 is the Grid Network To works considered in any test processed by processors which are placed in a grid network. For the same set of grid network model, called Model N_GMT is designed Where $\mathrm{N}$ represents the number $\mathrm{N}$ of Table 7, the model is designed to test the issue. And GMT geographical area intended for grid network show. Table 8 sets designed to model test data in Table 7 shows.

Table 8. Model set design

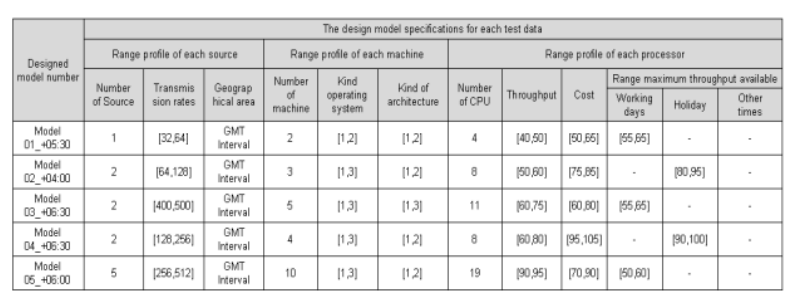

As shown in Table 8 for the total number of parameters designed to test the performance of the proposed algorithm is further studied. For example, in Table 7 on the grid system model, Test 01_H is designed with the Model 01_ +06:00, from Table 8 will be tested. 


\subsection{Results of the simulation}

In Table 9 the results of the proposed algorithm for testing data are shown in Table 7.

Table 9. Results of the proposed algorithm

\begin{tabular}{|c|c|c|c|c|c|c|c|c|}
\hline \multirow{2}{*}{ Test data } & \multicolumn{4}{|c|}{ Genetic Algorithms } & \multicolumn{4}{c|}{ Suggested algorithm } \\
\cline { 2 - 9 } & $\begin{array}{c}\text { Number of } \\
\text { generations }\end{array}$ & $\begin{array}{c}\text { Best } \\
\text { fithess }\end{array}$ & $\begin{array}{c}\text { Best } \\
\text { Runtme }\end{array}$ & $\begin{array}{c}\text { Best of } \\
\text { breed }\end{array}$ & $\begin{array}{c}\text { Number of } \\
\text { generations }\end{array}$ & $\begin{array}{c}\text { Best } \\
\text { finess }\end{array}$ & $\begin{array}{c}\text { Best } \\
\text { Runtime }\end{array}$ & $\begin{array}{c}\text { Best of } \\
\text { breed }\end{array}$ \\
\hline Test 01_W & 200 & $\begin{array}{r}645.80000 \\
5674362\end{array}$ & 476 & 190 & 200 & $\begin{array}{r}746.80000 \\
4005432\end{array}$ & 18 & 195 \\
\hline Test 02_H & 200 & $\begin{array}{r}253.00000 \\
3769994\end{array}$ & 288 & 126 & 200 & $\begin{array}{r}262.60000 \\
3913045\end{array}$ & 15 & 178 \\
\hline Test 03_W & 250 & $\begin{array}{c}317.00000 \\
4723668\end{array}$ & 365 & 126 & 250 & $\begin{array}{r}339.20000 \\
5054474\end{array}$ & 25 & 224 \\
\hline Test 04_H & 300 & $\begin{array}{r}285.60000 \\
6729364\end{array}$ & 778 & 219 & 300 & $\begin{array}{c}289.40000 \\
6830692\end{array}$ & 31 & 231 \\
\hline Test 05_W & 400 & $\begin{array}{r}592.49999 \\
6125698\end{array}$ & 2001 & 344 & 400 & $\begin{array}{r}599.39999 \\
5803833\end{array}$ & 91 & 374 \\
\hline
\end{tabular}

Figure 5 compares the two algorithms are fitness diagram shows the test data using the collection Test 02_H. As shown in Figure 5, the proposed algorithm is always better solutions than the algorithm in the GA.

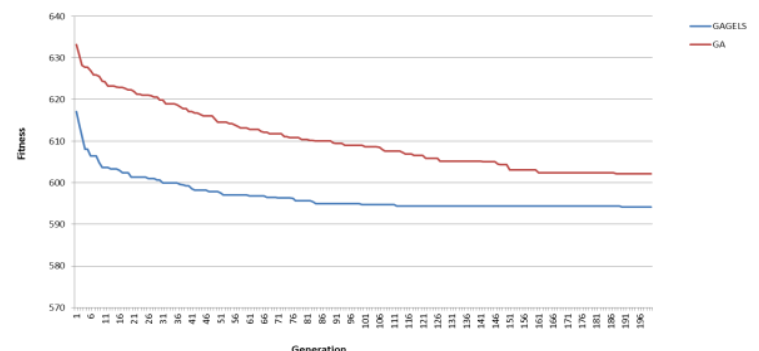

Figure 5. diagram comparing the fitness the proposed algorithm with GA Algorithms for Test Data Test 02_H

Figure 6, diagram fitness comparison algorithm using two test datasets Test 05_ W shows. As shown in Figure 6 is shown the proposed algorithm to test the algorithm, GA is always a better solution.

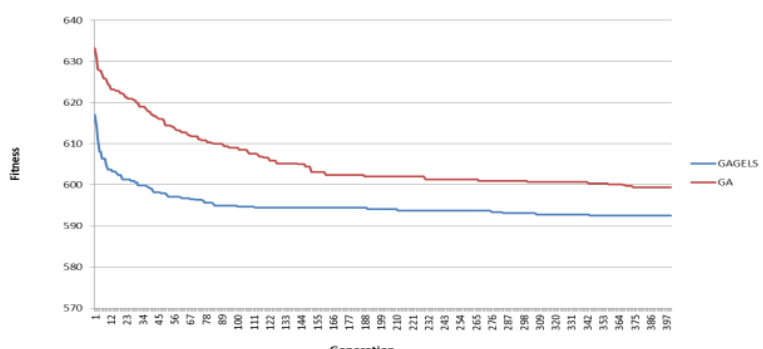

Figure 6. diagram comparing the fitness the two algorithms using datasets Test Test $05 \_W$

\section{Conclusion}

In this paper, a new method using gravity algorithm combines genetic algorithms and a system for grid scheduling problem is proposed. Proposed algorithm with genetic algorithm in powerful local search was combined gravitational force, the proposed algorithm is more efficient in achieving the optimum solution. It also has been trying to answer at the right time can achieve a higher quality. Eight different test data to evaluate the performance of the proposed algorithm, the proposed algorithm is designed for large and medium and small issues can be discussed. Experimental results show that the proposed algorithm in solving grid system has high speed and able to create a balance between users' criteria. Future work could affect the diversity of the population and prevent the convergence of the genetic algorithm on the solution quality of the proposed algorithm to solve the problem. Think of it as a priority for the allocation of resources to tasks.

\section{REFERENCES}

[1] Li. M., Baker, M. 2005, "the Grid, Core Technologies", John Wiley \& Sons Ltd.

[2] Jacob, Brown, M., Fukui, K., Trivedi, N. 2005 , "Introduction to Grid Computing", IBM.

[3] Garey, M. R. and Johnson, D. S. 1979, Computers and Intractability - A Guide to the Theory of NP Completeness,W. H. Freeman and Co.

[4] Wang, L., et al., et al. 1, s.1.1997, "Task matching and scheduling in heterogeneous computing environments using a genetic-algorithm-based approach": Journal of Parallel and Distributed Computing, Vol. 47, pp.8-22.

[5]. Braun, T. D., et al., et al. 6, s.1. 2001 ,"A comparison of eleven static heuristics for mapping a class of independent tasks onto heterogeneous distributed computing systems" : Journal of Parallel and Distributed Computing, Vol. 61, pp. 810-837.

[6] Carretero, J., Xhafa, F. and Abraham, A. s.l., 2007, "Genetic algorithm based schedulers for grid computing systems": International Journal of Innovative Computing, Information and Control, Vol. 3, pp. 10531071. 5

[7] Yuan, J. B., Luo, J. M. and Su, Z. Y. Wuhan, 2008, "Strategy for tasks scheduling in grid combined neighborhood search with improved adaptive genetic algorithm based on local convergence criterion". International Conference on Computer Science and Software Engineering. pp. 9-13.

[8] Fidanova, S. Sofia, Bulgaria,2006, "Simulated annealing for grid scheduling problem": IEEE John Vincent Atanasoff 2006 International Symposium on Modern Computing. pp. 41-45.

[9] Kazem, A. A. P., et al., et al. "A Modified Simulated Annealing Algorithm for Static Task Scheduling in Grid Computing". Singapore: International Conference on Computer Science and Information Technology. pp. 623627.

[10] Zheng, S. J., Shu, W. N. and Gao, L. Shanghai, 2006,"Task scheduling using parallel genetic simulated annealing algorithm", IEEE International Conference on Service Operations and Logistics, and Informatics (SOLI 2006), Vol. 1, pp. 46-50. 